\title{
Overview of dynamical mechanisms of secular evolution
}

\author{
Daniel Pfenniger
}

Geneva Observatory, Geneva, Switzerland

email: daniel.pfenniger@unige.ch

\begin{abstract}
Gravity-bound isolated systems, from stars, planetary systems, star clusters to galaxies, share common properties where evolution is the rule. Typically if they start forming at a well defined epoch they tend to change significantly over a timescale comparable to their present age. So evolution is never truly stopped, it just proceeds slower and slower: after a rapid, violent phase a slower, secular phase follows. In galactic astronomy for many decades the paradigm was rather that after a short violent time galaxies would settle in a stable steady state just consuming gas into stars. Actually today it appears that the progressive appearance of galaxy systematic morphologies and the slowing pace of mergers indicate that common intrinsic dynamical factors continue to shape galaxies towards similar properties irrespective of their largely different formation histories and initial conditions. Newtonian physics supplemented by a weakly dissipative component provides an amazing amount of explanations for the galaxy properties, like exponential stellar disks, spirals, bars, and peanut-shaped bulges. The purpose of this talk is to review these mechanisms of dynamical secular evolution.
\end{abstract}

Keywords. galaxies: evolution; galaxies: structure 